\title{
Exploiting Morpho-syntactic Features for Verb Sense Distinction in KorLex
}

\author{
Eunryoung Lee ${ }^{1}$, Ae-sun Yoon ${ }^{1}$, and Hyuk-Chul Kwon ${ }^{2}$ \\ ${ }^{1}$ Pusan National University, Korean Language Processing Laboratory \\ ${ }^{2}$ Pusan National University, Department of Computer Science and Engineering, \\ Jangjeon-dong, Geumjeong-gu, 609-735 Busan, S. Korea \\ \{eunryounglee, asyoon, hckwon\} @pusan.ac.kr
}

\begin{abstract}
Verb sense distinction is a basic principle in lexical knowledge representation in wordnets. Starting from the results of automatic mapping of English verb WordNet to Korean verb KorLex, the present study looks for a syntactic and semantic verb sense interface using the morpho-syntactic features of Korean verb and proposes a fine-grained verb sense distinction to make up for the weak points of WordNet in NLP applications.
\end{abstract}

Keywords: WordNet, KorLex, Korean wordnet, verb sense distinction, transitivity alternation, Korean middle verbs.

\section{Introduction}

A Korean verb wordnet named KoreLex was constructed based on the word-sense mapping of English WordNet (Princeton, version 2.0, hereafter WN) to Korean ${ }^{1}$ verbs. During semi-automatic translation, we noticed the following problems; (1) the English verbs of accusative(acc)/inaccusative(inacc) alternation were mapped to two or more morpho-syntactically different Korean lexical entities; (2) two English verb senses were matched to a Korean middle verb(MV), having both acc/inacc features but considered as having a unique sense in most Korean dictionaries. To cope with these problems, the verb sense distinction in $\operatorname{KorLex}(\mathrm{KL})$ should be processed primarily on the basis of coherent linguistic criteria. For this purpose, we explore morpho-syntactic features that are strikingly marked at the lexical level in Korean, and show how to apply them to KL's hierarchical structure.

The paper is structured as follows: Section 2 consists of an overview of related work in English and in Korean. In Section 3, we look at the translation results of 2,322 verb synonym sets (synsets) and analyze the types of mismatches. Section 4 proposes to allocate the distinguished verb senses according to their morpho-syntactic properties in the Korean verb hierarchy.

${ }^{1}$ Korean is an agglutinative language and the word order, as determined by case markers, is partially free. A verb's noun arguments, including the nominative and the accusative, can be omitted according to the context, and the verb is always located in the final position. Aspectual, modal markers as well as tense markers are postpositional morphemes agglutinated to the verb radical. 


\section{Related Work}

Effect of the syntactic features on the meaning of verbs has been the subject of theoretical debate in linguistics and it is still a controversial issue in building a lexical knowledge base or ontology. However, no concrete choice methodology, nor any theoretical settlement for the syntactic-semantic interface, for wordnet purposes, has been suggested. Languages differ in what syntactic features of verbs are encoded morphologically, and thus lexical formation and its morphological rules for certain syntactic phenomena should be considered as having a semantic effect, especially in agglutinative languages including Korean.

\subsection{Searching for Syntactic and Semantic Verb Sense Interface}

According to [3] and [5], the semantic information of a verb must include both its central meaning and the thematic grid it specifies, both of which determine the syntactic construction of the verb. Even though the more specific syntactic features and semantic role of English verbs are explored in the FrameNet and in VerbNet, it is very difficult to apply such information WN-like hierarchical semantic network.

Previous studies on WN's verb sense distinction have attempted to complete WN by integrating the syntactic properties into the verbs in order to extend the lexical information. Consequently, questions regarding the means by which syntactic information in a verb lexicon are arranged, and by which verb senses are distinguished, are important issues. In this respect, grouping about 3,000 English verbs according to diathesis alternation patterns, [9] is a pioneering work which led also to a set of studies on verb polysemy.

Among a number of extensive studies on Levin's work, [7] and [12] identifies missing verb senses in transitive alternation and testing accuracy by mapping Levin's classification to WN. Although current researchers in the field of lexical semantics have concluded that certain syntactic behavior traits of verbs are central to their meaning, it has not been clearly established that the diathesis alternation results in a change of meaning. Regarding this subject, [10] argues that case-alternated verbs should be considered logically as polysemous and that therefore Levin's verb class can be used for verb sense disambiguation.

\subsection{Case Alternation in Korean}

Case alternation in Korean is characterized by (a) the position change of a noun argument accompanying a change of its semantic role, (b) the reduction/ extension of number of the noun argument, and (c) alternation of the case marker. It is generally accepted that the case in Korean is determined by the case marker and according to [4], case does not have the same grammatical properties as in English. [7] claims that since case alternation has to be described additionally according to the semantic 
property of a noun argument at the phrasal level, it is difficult to consider that the meaning of a verb changes according to its case alternation. In this respect, the MV in Korean might offer a theoretical basis for verb sense distinction in a wordnet; the verb allows both $a c c$ and inacc frames on a verb radical and the acc noun argument in the transitive frame can be alternatively, be the nominative noun in the intransitive frame. Two syntactic realizations of the MV "baljok-hada (launch)" allow two different senses, as in following example.

(1) keu-neun sailoun hakheoi-leul baljogha-yeoss-da

He-nominative new academic society-acc launch-past-final

(He launched a new academic society.)

sailoun hakheoi-ga
new society-nominative $\frac{\text { baljogha-yeoss-da }}{\text { launch-past-final }}$
(A new academic society was launched.)

In the acc frame, the MV has a causative synonymous form, and in the inacc frame, it is passive. At this point, it is useful to explore the sense distinction of MVs to be sub-categorized both for the lexicographical purpose and for a WN-like linguistic NLP application resource.

\section{Verb Sense Distinction for KorLex Verb}

Semi-automatic translation using bilingual dictionary does not fully reflect the meanings of English verbs in Korean verbs when they contain both acc and inacc frames at the same time. It is remarkable that pairs of transitive/intransitive verbs and transitive/passive verbs are subject to more refined sense distinction ${ }^{2}$ and modification of hierarchical structure in KL. In KL, a synset is defined as a set of synonymous verb senses that should be interchangeable in least one context, which means that the syntactic behavior of a verb is considered to be the core element determining its semantic relations in KL.

\subsection{Verb Sense Linking}

Here, we will examine the Korean counterparts of English \{verbs.change\} and analyze the mapping result in accordance with transitivity alternation. As for the translation process between the two languages, we first mapped 2,325 English synsets to Korean verbs using an English-Korean dictionary, obtaining 3,184 senses. The automatic mapping process failed to provide a full and correct translation into Korean, thus manual verification and correction were necessary.

We chose only 972 English-Korean verb synsets (corresponding to 1,314 verb senses). We did not take into consideration the rest of the 1,353 synsets, translated into verb phrases. Therefore, for the Korean verbs, we selected 972 synsets

\footnotetext{
${ }^{2}$ See [8] for the sense distinction in WN and KL.
} 
(1,344 verb senses) including only those appearing as entries in the Standard Korean Dictionary ${ }^{3}$. The following Figure illustrates the mapping result.

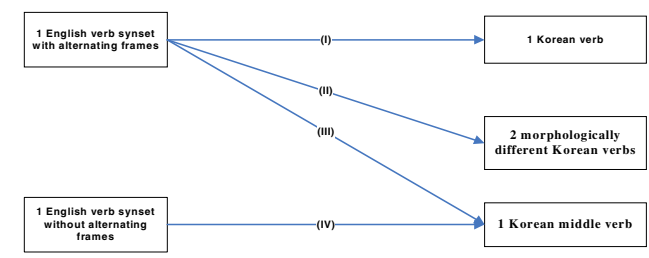

Fig. 1. Mapping matrix for English and Korean verbs

As shown in Fig.1, we find four types of mapping result. The two left boxes indicate the syntactic status of the English verb synset; the upper box indicates the verbs with acc and inacc frames. The lower box indicates the verbs having no alternating frames.

Type (I) is the most common in our data, numbering up to 1,028 Korean verb senses among which 129 verb senses were found to be erroneous. Wrong connections and missing word senses were post-processed manually. After correction, since an English verb has two frames, the matching Korean verb set contains two morpholexically different verbs. Thus the Korean verb sets found in Type (I) are subject to the sense distinction.

According to Type (II), English synsets are mapped to two different verb senses that are either transitive-intransitive (for 65 English synsets), or transitive-passive pairs (for 34 English synsets). To check the translation accuracy for Type (II), we examined whether the gloss of an English synset contained more than two senses. It appears that in most cases the sentence frames distinguish the acc/inacc senses more accurately rather than the gloss. The Korean verb sets in this type ultimately need to be separated into an independent synsets.

According to Type (III), English transitivity-alternated verbs are mapped to a Korean MV also having two alternating frames according to transitivity alternation. However, only 8 Korean MVs were matched. Regarding Type (III), it is necessary that the refinement of translation results goes through multiple post-processing. Finally, according to Type (IV), 50 Korean MVs are matched to either English acc or inacc form, which process reflects the necessity of verb sense distinction of Korean MVs. In the following two subsections, 3.2 and 3.3, we discuss the necessity of modifying verb sense distinction in KL focusing on Type (II) and Type (III).

\subsection{Sense Distinction: Transitive/Passive Form}

According to Type (II), one English verb synset having alternating frames is mapped to either a transitive/intransitive verb pair (II-a) or a transitive/passive verb pair (II-b).

\footnotetext{
${ }^{3}$ We used the Standard Korean Dictionary, which contains 58,815 Korean verb entries. English-Korean bilingual dictionary we used contains 14,454 verb entries.
} 
A translated set of verbs is subject to sense distinction in KL for two reasons, as shown in the following examples.

(2) Na-neun gamja-leul jiji-n-da

I-nominative potato-acc steam-present-final

(I steam the potatoes.)

(3) Gamja-neun soss-eseo bbali jjyeo-ji-n-da

Potato-nominative pot-locative rapidly steam-passive-present-final

(The potato steams rapidly in the pot.)

First, "jji-da" to "jjy-eoji-da," have different selectional restrictions and syntactic distributions. The passivization of "jji-da" is realized by "-eojida" which is an auxiliary verb. A passive verb in Korean is derived by postpositional morphemes in the following three ways; (1) adhesion of a suffix (2) for verbs such as $<$ noun+hada(support verb) $>$, adhesion of morphemes such as -deo, -bad, or -dangha; (3) adhesion of the auxiliary verb -jida. In Korean by contrast to English, the passive voice does not necessarily presuppose a syntactic alternation, but it is realized at the lexical level by the suffixation of a specific morpheme. Providing ten passive sentence structure, [11] proves that the Korean passivisation is not a syntax-dependent process and that it is rather a morpho-semantic feature.

Second, following the WN's basic assumption, it is not possible in Korean to put those verb forms in a synset. A synset is defined as a set of synonymous words that are interchangeable in some context. But the Korean passive and transitive verb forms cannot be substituted in any context.

\subsection{Sense Distinction for Korean Middle Verbs}

In Figure 1, Type (III) corresponds to the mapping between an English synset with two alternating frames and a Korean synset with a MV. We believe that in KL, MVs should be treated differently from the way they are treated in English WN.

As shown in Example (1), Subsection 2.2, the distributional trait of a Korean MV provides a syntactic basis for semantic distinction. In addition, we think that semantic features, even though they are not necessary and sufficient conditions for all MVs, are inherent to MVs, and thus can provide linguistically positive arguments for the polysemy of the MV, because (1) the acc frame of a MV is semantically identical to its paraphrased sentence with a causative verb, and (2) the inacc frame of a MV is semantically identical to its paraphrased sentence with a passive verb, especially when the noun argument in the nominative case does not assume the agentive. The disparity of semantic properties of MVs in acc/inacc constructions has not been suggested in Korean linguistics as an argument for their sense distinction, since they are used as additional criteria for identifying, on the syntactic basis, middle constructions from other types of transitivity alternation. However, we argue that the sense of a MV varies according to the syntactic construction and that different semantic relations such as in the synonymy of a MV in two constructions are footholds for sense distinction in KL Verb. 


\section{Building KorLex Verb Hierarchy}

This section is devoted to the actual building of a KL verb hierarchy by applying the verb sense distinction suggested in Subsections 3.2 and 3.3. Verb hierarchy in KL is constituted with the troponymy(hyponym) relation which should be adequate for representing the change of meaning according to the syntactic construction, allowing for a fine-grained sense distinction useful to sentence parsing in NLP applications.

\subsection{Passive Verb Classification and Its Place in KorLex}

In KL, since verb hierarchy is biased according to the WN structure, it is efficient to look for the appropriate place in relation to the top-hypernyms and their hyponyms in WN. In Table 1, the top-hypernyms of Type A (pairs of acc and inacc concepts) reflect the acc/inacc values independently; \{change 0$\}$ is inacc and \{change1, alter1, modify 11 \}, accusative. Given the 35 top hypernyms of \{verb.change\}, we can choose an appropriate place in the hierarchies for the Korean passive or transitive verbs by following their syntactic and semantic features. The following Figure 2 represents the hierarchical structure of $\{$ bake 0$\}$.

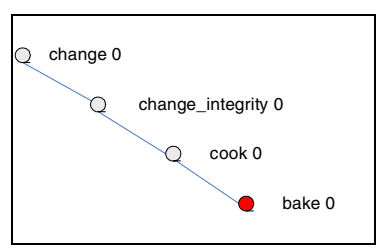

Fig. 2. Hierarchy of $\{$ bake 0$\}$

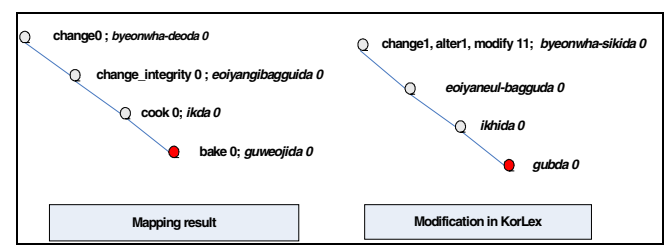

Fig. 3. Reconstruction of hierarchy for Passive/Transitive verb "gubda"

In the hierarchy of $\{$ bake 0$\}$, hypernyms are constituted of verb senses of inacc frame. Thus we will use this structure to match the passive verb "gu-weoji-da" (bakepassive-final), and the Korean counterparts for the hypernyms of $\{$ bake 0$\}$ will be all of passive value. Now, we can separately build the transitive and the passive forms of "gub-da"(bake-final) as shown in Figure 3. The structure on the left is the hierarchy of the passive form of the verb "guweojida" and its hypernyms of passive value. The structure on the right represents the modified hierarchy of the transitive verb form and its hypernyms of transitive value. Each counterpart of \{bake 0$\}$ now has a different semantic relation in KL. As a result, \{bake 0$\}$ is mapped accurately to Korean synonym "guweojida" and \{cook 0$\}$ is mapped to "ikda". The hierarchy on the right also shows the additional nodes by which "gubda" is represented accusatively.

\subsection{Middle Verb Sense Distinction and Its Place in KorLex}

KL requires three different processes of MV sense distinction:

(1) separate the two senses of a $M V$ and add them to appropriate existing $K L$ synset

(2) separate the two senses and create a node for a new sense in the KL hirerarchy

(3) separate the two senses and create both a node and a new hierarchy in KL 
Process (1) is applied to verbs for which we can find an appropriate synset for the two distinguished KL senses. For example, the sense of the MV 'gwayeolhada (overheat)' was separated into 'gwayeolhada 0 (be overheated)' / 'gwayeolhada1 (overheat)' and added as a synonym to the existing synset node in KL, as shown in Figure 4.

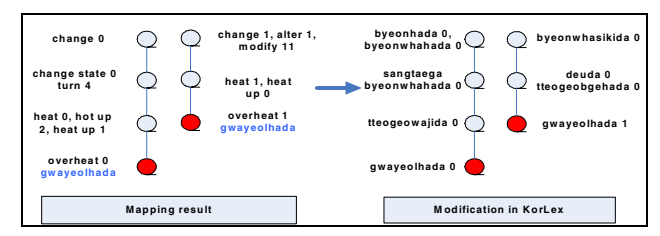

Fig. 4. Sense distinction and position of 'gwayeolhada' in KL

Process (2) was applied, for example, to the MV 'baigahada (multiply)'.

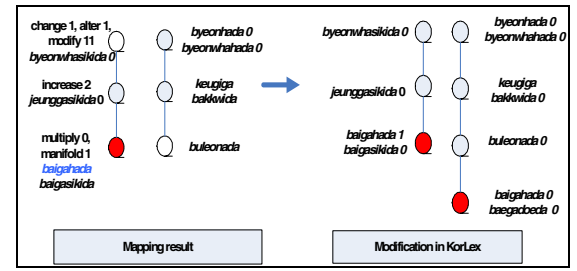

Fig. 5. Sense distinction of 'baigahada' in KL

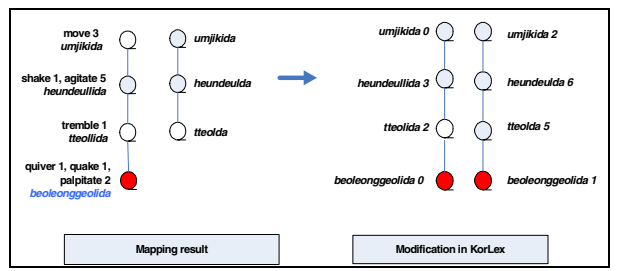

Fig. 6. Sense distinction o 'beoleonggeolida' in $\mathrm{KL}$

In the mapping result, 'baigahada (multiply)' was mapped to the English synset \{multiply 0 , manifold 1 \}, also subject to sense distinction. We distinguished 'baigahada (multiply)' according to 'baigahada 0 (be multiplied)' and 'baigahada 1 (multiply).' 'baigahada 0' was linked to \{multiply 0, manifold 1 \}, and the new sense 'baigahada 1' was created as a troponym of 'buleonada 2 as shown in Figure 5. Process (3) is the same process but with the addition to KL of a new Korean verb sense. Fig 6 shows process (3) applied to 'beoleonggeolida (shake vigorously).' In consideration of the hypernyms, its sense has to be of inacc value. Therefore, after the sense distinction of 'beoleonggeolida' according to 'beoleonggeolida 0 (be shaken vigorously) and 'beoleonggeolida 1 (shake vigorously),' the latter requires a new set of hypernymys, which is also added to KL.

\section{Conclusion}

Semi-automatic construction of KL using WN as pivot requires elaborate linguistic criteria determining the grain size of sense and semantic relation. KL verb is constructed based on English WN, but its semantic relations and sense distinctions are modified. In this study, focusing on the relatedness of morpho-syntactic features and the verb meanings, we showed that the sense distinction can be manageable by 
extracting a different pair of syntactic frames - acc/inacc - for polysemous verbs in Korean. Moreover, we demonstrated that the morpho-syntactic-based sense distinction can be applied in establishing the semantic relation of hypernymytroponymy, which has been considered difficult to achieve. We think that this is useful to any verb wordnet applied to NLP.

The problem of sense distinction is directly connected to the question of polysemy and word sense disambiguation. However, for verb sense distinction, linguistically robust criteria are necessary with or without limitation of generalization, since there is no single method applicable to all lexical acquisitions.

\section{Acknowledgment}

This work was supported by the Korea Science and Engineering Foundation (KOSEF) through the National Research Lab. Program funded by the Ministry of Science and Technology (No. M10400000332-06J0000-33210).

\section{References}

1. Cruse, D.A.: Lexical Semantics. Cambridge University Press, Cambridge (1986)

2. Fellbaum, C.: WordNet- An Electronic Lexical Database. MIT Press, Cambridge (1998)

3. Grimshaw, J.: Argument Structure. MIT Press, Cambridge (1990)

4. Hong, J.S.: Nouns in Korean: Sai Kukeosaingwhal, Natioanl Institut of the Korean Language vol.11-3, Seoul (2001), html version

5. Jackendoff, R.S.: Semantic Structures. MIT Press, Cambridge (1990)

6. Kim, M.L.: The Studies of Verbal Classes according to Case Mark-Alternation: Korean Linguistics vol.25, Association for Korean Linguistics, Seoul (2004) 161-190

7. Kohl. T., Jones, D.A., Berwick, R.C. and Nomura, N.: Representing Verb Alternation in WordNet: WordNet- An Electronical Lexical Database. MIT Press, Cambridge(1998) 153-178

8. Lee, E-R, Yoon, A-S and Kwon, H-C: Passive Verb Sense Distinction in Korean Wordnet: Proceedings of the $3^{\text {rd }}$ International WordNet Conference, GWC, Brno (2006) 211-216

9. Levin, B.: English Verb Classes and Alternations-A Preliminary Investigation. University of Chicago Press, Chicago (1993)

10. Pustejovsky, J.: The Generative Lexicon. MIT Press, Cambridge, London (1998)

11. Yang, J-S: Semantic Analysis of Korean Verb and Linking Theory. Bakijeong, Seoul (1995)

12. Zickus, W. M.: A Comparative Analysis of Beth Levin's English Verb Class Alternations and WordNet's Senses for the Verb Classes HIT, TOUCH, BREAK, and CUT.: Proceedings of The Post-Coling 94 International Workshop on Directions of Lexical Research. Beijing, China: Tsinghua University (1994) 66-74 